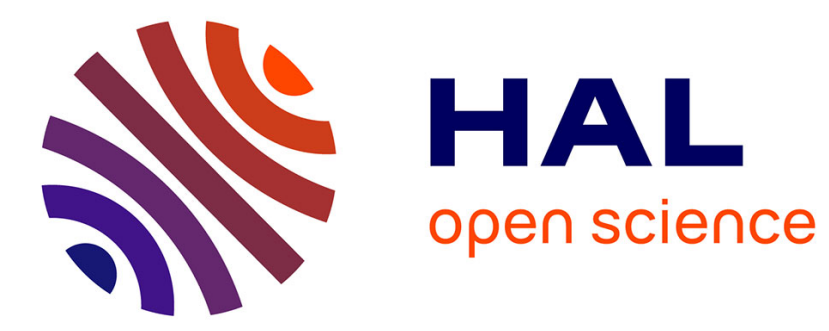

\title{
Decay of solutions of wave equation in a star-shaped domain with nonlinear boundary feedback
}

\author{
Francis Conrad, Bopeng Rao
}

\section{To cite this version:}

Francis Conrad, Bopeng Rao. Decay of solutions of wave equation in a star-shaped domain with nonlinear boundary feedback. [Research Report] RR-1381, INRIA. 1991, pp.28. inria-00075180

\section{HAL Id: inria-00075180 \\ https://hal.inria.fr/inria-00075180}

Submitted on 24 May 2006

HAL is a multi-disciplinary open access archive for the deposit and dissemination of scientific research documents, whether they are published or not. The documents may come from teaching and research institutions in France or abroad, or from public or private research centers.
L'archive ouverte pluridisciplinaire HAL, est destinée au dépôt et à la diffusion de documents scientifiques de niveau recherche, publiés ou non, émanant des établissements d'enseignement et de recherche français ou étrangers, des laboratoires publics ou privés. 


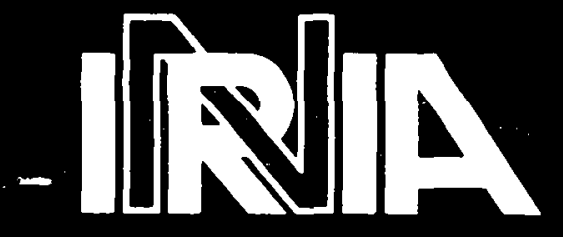

UNITÉ DE RECHERCHE INRIA-LORRAINE

s

Institut National de Recherche en Informatique et en Automatique

Domaine de Voluceau - Rocquencourt

$$
\text { B.P.105 }
$$

78153 Le Chesnay Cedex

$$
\text { France }
$$

Tél.:(1)39635511

\section{Rapports de Recherche}

$$
N^{\circ} 1381
$$

\section{Programme 6}

Calcul scientifique, Modélisation et

Logiciels numériques

DECAY OF SOLUTIONS OF WAVE EQUATION IN A STAR-SHAPED DOMAIN WITH NONLINEAR BOUNDARY FEEDBACK

\section{Francis CONRAD}

Bopeng RAO

Janvier 1991 


\title{
Decay of Solutions of Wave Equation in a Star-Shaped Domain with Nonlinear Boundary Feedback
}

\author{
Francis CONRAD *, Bopeng RAO *
}

\begin{abstract}
We study the uniform stabilization of the wave equation by means of a nonlinear dissipative boundary feedback. We consider a Neumann condition on the whole boundary, and the observation is the boundary displacement and velocity. Extending a result of E. Zuazua, we obtain, in a nonlinear framework, estimates of the decay, for any displacement. We establish a similar result for the one-dimensional wave equation with a variable coefficient.
\end{abstract}

\section{Stabilisation de l'Equation des Ondes dans un Domaine Etoilé au Moyen d'un Contrôle Frontière non Linéaire}

\section{Résumé}

On étudie la stabilisation uniforme de l'équation des ondes au moyen d'un feedback frontière dissipatif non linéaire. On considère une condition de Neumann sur tout le bord, l'observation étant la vitesse et le déplacement. On généralise un résultat de $\mathrm{E}$. Zuazua, en obtenant, dans le cadre non linéaire, des estimations de décroissance quelle que soit l'amplitude du déplacement. On établit un résultat analogue pour l'équation des ondes monodimensionnelle, à coefficient variable.

(*)Université de Nancy I, U.R.A. CNRS 0750 and Projet Numath, INRIA Lorraine Département de Mathématiques, B.P. 239, 54506 VANDOEUVRE-lès-NANCY, France. 


\section{Introduction}

Let $\Omega$ be a bounded domain of $\mathbb{R}^{n}$, strictly star-shaped with respect to $x_{0} \in \Omega$ and having smooth boundary $\Gamma=\partial \Omega$ of class $C^{2}$. We define the mapping $m: \mathbb{R}^{\mathbf{n}} \longrightarrow \mathbb{R}^{\mathbf{n}}$ by

$$
\mathrm{m}(\mathrm{x})=\mathrm{x}-\mathrm{x}^{0} \text {. }
$$

For all $x \in \Gamma$, we have $m(x) \cdot v(x)>0$, where $v(x)$ is the outer unit normal vector to $\Gamma$ at $x \in \Gamma$, and ". " denotes the scalar product in $\mathbb{R}^{\mathrm{n}}$. Since $\Omega$ is bounded with smooth boundary $\Gamma$, there is a positive constant $\delta>0$, suth that

$$
\mathrm{m}(\mathrm{x}) \cdot \mathrm{v}(\mathrm{x})>\delta^{-1}, \quad \forall \mathrm{x} \in \Gamma \text {. }
$$

We denote by $\frac{\partial}{\partial v}$ the normal derivative and by " ' " the time-derivative $\frac{\mathrm{d}}{\mathrm{dt}}$.

Let us consider the following wave equation

$$
\begin{array}{lc}
y^{\prime \prime}-\Delta y=0 & \text { in } \Omega \times(0, \infty) \\
\frac{\partial y}{\partial \nu}+\alpha y=-g\left(y^{\prime}\right) & \text { on } \Gamma \times(0, \infty) \\
y(0)=y_{0} \in H^{1}(\Omega), y^{\prime}(0)=y_{1} \in L^{2}(\Omega)
\end{array}
$$

where $\alpha \in \mathrm{L}^{\infty}(\Gamma)$ satisfies $\alpha(\mathrm{x}) \geq \alpha_{0}>0, \forall \mathrm{x} \in \Gamma$, and $\mathrm{g}: \mathbb{R} \longrightarrow \mathbb{R}$ is a continuous nondecreasing function such that

$$
g(0)=0, g(s) s>0,|g(s)| \leq C(1+|s|), \text { for all } s \neq 0 \text {. }
$$

It is well-known that for any initial data $\left(y_{0}, y_{1}\right) \in H^{1}(\Omega) \times L^{2}(\Omega)$, the equations (1.3)(1.5) have a unique weak solution y such that

$$
y \in \mathrm{C}^{0}\left(\mathbb{R}^{+}, \mathrm{H}^{1}(\Omega)\right) \cap \mathrm{C}^{1}\left(\mathbb{R}^{+}, \mathrm{L}^{2}(\Omega)\right) .
$$

We define the energy associated with a solution y of the equations (1.3)-(1.5) as follows:

$$
E(t)=\frac{1}{2} \int_{\Omega}\left(|\nabla y|^{2}+\left|y^{\prime}\right|^{2}\right) d x+\frac{1}{2} \int_{\Gamma} \alpha y^{2} d \Gamma .
$$

By Green's formula, we obtain (at least formally at this stage)

$$
\frac{d}{d t} E(t)=E^{\prime}(t)=-\int_{\Gamma} g\left(y^{\prime}\right) y^{\prime} d \Gamma \leq 0
$$


The assumption (1.6) implies that the energy $E(t)$ is nonincreasing and defines a Lyapunov function. It is easy to prove the strong stabilization by applying Lasalle's invariance principle (see [5], [17] ). Indeed, in the more general cases for which $g$ is a maximal monotone graph, the strong asymptotic stabilization has also been proved ([4], [13], [14]). Our aim in the present paper is to estimate the rate of decay of the energy $E(t)$, when the nonlinear feedback function $g$ satisfies suitable growth conditions.

The problem of estimating the rate of decay of the energy $E(t)$ has been studied extensively by many authors, among which we mention Lagnese and Chen. Recently, Zuazua [19] using a method of multipliers, established estimates in the case where $\alpha>0$ is a small constant. In the case of a linear feedback, he also proved the uniform exponential decay for any $\alpha>0$ by a compactness-uniqueness argument [18] (see also [12]).

We obtain here an improvement of these results in the sense that we prove the estimates for any positive $\alpha \in \mathrm{L}^{\infty}(\Gamma)$ in the case of nonlinear feedback $\mathrm{g}$. Also, in the one dimensional case, we establish the estimates for the wave equation with variable coefficient.

Our two main results are as follows:

Theorem 1. Let $\Omega$ be a bounded domain of $\mathbb{R}^{\mathrm{n}}$ strictly star-shaped with respect to $\mathrm{x}_{0} \in \Omega$ and having smooth boundary $\Gamma=\partial \Omega$ of class $C^{2}$. Assume that $\mathrm{g}$ is a continuous nondecreasing function such that (1.6) holds. Then for every solution y of the equations (1.3)(1.5), we have:

(i) If there exist some positive constants $C_{1}, C_{2}$ such that

$$
\mathrm{C}_{1}|\mathrm{~s}| \leq|\mathrm{g}(\mathrm{s})| \leq \mathrm{C}_{2}|\mathrm{~s}|, \quad \forall \mathrm{s} \in \mathbb{R}
$$

then given any constant $M>1$, there exists a constant $\lambda>0$ such that (1.11) $\quad E(t) \leq M E(0) e^{-\lambda t}, \forall t \geq 0$.

(ii) If there exist some positive constants $C_{1}, C_{2}$ and $p>1$ such that

$$
\mathrm{C}_{1} \operatorname{Min}(|\mathrm{s}|,|\mathrm{s}| \mathrm{p}) \leq|\mathrm{g}(\mathrm{s})| \leq \mathrm{C}_{2}|\mathrm{~s}|, \quad \forall \mathrm{s} \in \mathbb{R}
$$

then given any constant $M>1$, there exists a constant $\mu>0$ depending on $E(0)$ such that

$$
\mathrm{E}(\mathrm{t}) \leq \mathrm{ME}(0)(1+\mu \mathrm{t})^{\frac{-2}{\mathrm{p}-1}}, \quad \forall \mathrm{t} \geq 0 .
$$


Theorem 2. Let $\Omega$ be a bounded domain of $\mathbb{R}^{n}$ strictly star-shaped with respect to $x_{0} \in \Omega$ and having smooth boundary $\Gamma=\partial \Omega$ of class $C^{2}$. Assume that $g$ is a continuous nondecreasing function such that (1.6) holds. Let $y$ be any solution of the equations (1.3) (1.5). If there exist some positive constants $C_{1}, C_{2}$ and $p<1$ such that

$$
\mathrm{C}_{1}|\mathrm{~s}| \leq|\mathrm{g}(\mathrm{s})| \leq \mathrm{C}_{2} \operatorname{Max}(|\mathrm{s}|,|\mathrm{s}| \mathrm{p}), \quad \forall \mathrm{s} \in \mathbb{R}
$$

then given any constant $M>1$, there exists a constant $\mu>0$ depending on $E(0)$ such that

$$
\mathrm{E}(\mathrm{t}) \leq \mathrm{ME}(0)(1+\mu \mathrm{t})^{\frac{-2 \mathrm{p}}{1-\mathrm{p}}}, \quad \forall \mathrm{t} \geq 0
$$




\section{Proof of the main results}

For the sake of completeness, we start with a brief outline of the well-posedness of the equations (1.3)-(1.5) by the semi-group approach.

Let us define

$$
H=H^{1}(\Omega) \times L^{2}(\Omega)
$$

with the scalar product

$$
<(y, \hat{y}),(z, \hat{z})>=\int_{\Omega}(\nabla y \nabla z+\hat{y} \hat{z}) d x+\int_{\Gamma} \alpha y z d \Gamma
$$

We introduce the nonlinear operator $\mathrm{A}$ on $\mathrm{H}$ :

$$
\mathrm{D}(\mathrm{A})=\left\{(\mathrm{y}, \hat{\mathrm{y}}) \in \mathrm{H}^{1}(\Omega) \times \mathrm{H}^{1}(\Omega) ; \Delta \mathrm{y} \in \mathrm{L}^{2}(\Omega) ; \frac{\partial \mathrm{y}}{\partial v}+\alpha \mathrm{y}=-\mathrm{g}(\hat{\mathrm{y}}) \text { on } \Gamma\right\}
$$

which is dense in $H$, and for any $(y, \hat{y}) \in D(A)$,

$$
A(y, \hat{y})=(-\hat{y},-\Delta y)
$$

Remark 2.1 Here $\Delta y$ is defined in the sense of distributions. We see that the normal derivative $\frac{\partial y}{\partial v}$ is not defined by the usual trace theorem. The equality $\frac{\partial y}{\partial v}+\alpha y=-g(\hat{y})$ on $\Gamma$ means that

$$
\int_{\Omega} \Delta \mathrm{y} \phi \mathrm{dx}+\int_{\Omega} \nabla \mathrm{y} \nabla \phi \mathrm{dx}+\int_{\Gamma} \alpha \mathrm{y} \phi \mathrm{d} \Gamma+\int_{\Gamma} \mathrm{g}(\hat{\mathrm{y}}) \phi \mathrm{d} \Gamma=0, \forall \phi \in \mathrm{H}^{1}(\Omega) .
$$

However, when both definitions of the traces make sense, they coincide.

By means of the operator $\mathrm{A}$, we transform the equations (1.3)-(1.5) into the following operational form:

$$
(y, \hat{y})^{\prime}+A(y, \hat{y})=0, \quad(y(0), \hat{y}(0))=\left(y_{0}, y_{1}\right) \in H
$$


It is easy to prove that $\mathrm{A}$ is a maximal monotone operator on $\mathrm{H}$. Using the methods of Brezis [2], Haraux [8] or Lions and Magenes [16], we deduce that for any initial data $\left(y_{0}, y_{1}\right)$ $\in D(A)$, the equation (2.6) has a unique strong solution y such that

$$
\begin{aligned}
& y \in W^{1, \infty}\left(\mathbb{R}^{+}, H^{1}(\Omega)\right), \quad \Delta y \in L^{\infty}\left(\mathbb{R}^{+}, L^{2}(\Omega)\right), \\
& \|(y(t), \hat{y}(t))\|_{H} \leq\left\|\left(y_{0}, y_{1}\right)\right\|_{H}, \quad \forall t \geq 0 .
\end{aligned}
$$

Furthermore, since $D(A)$ is dense in $H$ for the strong topology, we can extend the mapping

$$
\left(y_{0}, y_{1}\right) \rightarrow(y(t), \hat{y}(t))
$$

to a strongly continuous semi-group of contractions $(S(t))_{\geq 0}$ in $\mathrm{H}$. Thus for every initial data $\left(y_{0}, y_{1}\right) \in H$, we can define the unique weak solution of the equation (2.6) by the following formula:

$$
(y(t), \hat{y}(t))=S(t)\left(y_{0}, y_{1}\right), \quad \forall t \geq 0
$$

with the regularity

$$
y \in C^{0}\left(\mathbb{R}^{+}, H^{1}(\Omega)\right) \cap C^{1}\left(\mathbb{R}^{+}, L^{2}(\Omega)\right) .
$$

By the density of $D(A)$ in $H$ and the fact that $(S(t))_{t \geq 0}$ is a strongly continuous semigroup of contractions in $\mathrm{H}$, it is sufficient to prove Theorems 1 and 2 for smooth initial data $\left(y_{0}, y_{1}\right) \in D(A)$. Therefore we assume in the sequel that the regularity (2.7) holds.

Let us recall some technical results:

Lemma 2.2 Let $\Omega$ be a bounded domain of $\mathbb{R}^{n}$, with smooth boundary $\Gamma=\partial \Omega$ of class $C^{2}$. Then there exists a constant $\beta>0$ suth that for any $y \in H^{1}(\Omega)$, the solution $\phi$ of the equation

$$
\begin{cases}-\Delta \varphi=0 & \text { in } \Omega, \\ \varphi=y & \text { on } \Gamma,\end{cases}
$$

satisfies the following estimates: 


$$
\int_{\Omega}|\varphi|^{2} \mathrm{dx} \leq \beta^{2} \int_{\Gamma}|\mathrm{y}|^{2} \mathrm{~d} \Gamma
$$

$$
\int_{\Omega} \nabla \varphi \nabla \mathrm{ydx} \geq 0
$$

Proof. Inequality (2.13) is standard in the theory of elliptic equations. In order to prove (2.14), it is enough to multiply (2.12) respectively, by $\varphi$ and y. By comparison, it follows that

$$
\int_{\Omega} \nabla \varphi \nabla y \mathrm{dx}=\int_{\Omega}|\nabla \varphi|^{2} \mathrm{dx}
$$

Remark 2.3 It is clear that problem (2.12) is equivalent to the following minimization problem:

$$
|\nabla \varphi|_{L^{2}(\Omega)}=\operatorname{Inf}_{\substack{\psi \in H^{1}(\Omega) \\ \psi=y \text { on } \Gamma}}|\nabla \psi|_{L^{2}(\Omega)}
$$

So $\varphi$ is the function which takes the same values as $y$ on the boundary $\Gamma$ and has least norm $|\nabla \varphi|_{L} 2_{(\Omega)}$. This function plays an important role in the proof of Theorem 1 (see Remark 2.6).

Lemma 2.4 Let $\Omega$ be a bounded domain of $\mathbb{R}^{\mathrm{n}}$ strictly star-shaped with respect to $\mathrm{x}_{0} \in \Omega$ and having smooth boundary $\Gamma=\partial \Omega$ of class $C^{2}$. Then the following inequality holds:

$$
2 \int_{\Omega} \Delta \mathrm{y}(\mathrm{m} \cdot \nabla \mathrm{y}) \mathrm{dx} \leq(\mathrm{n}-2) \int_{\Omega}|\nabla \mathrm{y}|^{2} \mathrm{dx}+\mathrm{R}^{2} \delta \int_{\Gamma}|\mathrm{v}| 2 \mathrm{~d} \Gamma
$$

for any function $y \in H^{1}(\Omega)$ such that $\Delta y \in L^{2}(\Omega)$ and $\frac{\partial y}{\partial v}=v \in L^{2}(\Gamma)$. In $(2.16)$, we have set $R$ $=\left\|_{\mathrm{m}}\right\|^{\infty}(\Gamma)^{\cdot}$

Proof. First, we assume $\frac{\partial y}{\partial v}=v \in H^{\frac{1}{2}}(\Gamma)$. In this case, we know that $y \in H^{2}(\Omega)$, and the following identity due to Rellich (see for instance [12]) holds :

$$
2 \int_{\Omega} \Delta y(m \cdot \nabla y) d x=(n-2) \int_{\Omega}|\nabla y| 2 d x
$$




$$
+2 \int_{\Gamma} \frac{\partial y}{\partial v}(\mathrm{~m} . \nabla \mathrm{y}) \mathrm{d} \Gamma-\int_{\Gamma}(\mathrm{m} . \mathrm{v})|\nabla \mathrm{y}| 2 \mathrm{~d} \Gamma, \text { for all } \mathrm{y} \in \mathrm{H}^{2}(\Omega) .
$$

By the geometrical condition (1.2), we deduce from (2.17) and Young's inequality applied to $\int_{\Gamma}^{\partial y} \frac{\partial \mathrm{m} \cdot \nabla \mathrm{y}) \mathrm{d} \Gamma \text { that }}{\partial v}$

$$
2 \int_{\Omega} \Delta y(m \cdot \nabla y) d x \leq(n-2) \int_{\Omega}|\nabla y|^{2} d x+R^{2} \delta \int_{\Gamma}|v| 2 d \Gamma \text { for all } y \in H^{2}(\Omega) .
$$

Now, consider $v \in L^{2}(\Gamma)$. By density, there exists a sequence $v_{k} \in H^{\frac{1}{2}}(\Gamma)$ such that $v_{k} \rightarrow v$ in $\mathrm{L}^{2}(\Gamma)$. Let $\mathrm{y}_{\mathrm{k}}$ be the solution of the equation

$$
\begin{cases}y_{k}-\Delta y_{k}=y-\Delta y & \text { in } \Omega \\ \frac{\partial y_{k}}{\partial v}=v_{k} & \text { on } \Gamma .\end{cases}
$$

Then (2.18) is valid for $\left(v_{k}, y_{k}\right)$. By the classical regularity theory, we know that $\left\|y-y_{k}\right\|_{H^{1}(\Omega)} \leq C\left\|_{v}-v_{k}\right\|_{H^{-1 / 2}(\Gamma)}$. So $y_{k} \rightarrow y$ in $H^{1}(\Omega)$ and since $\Delta y_{k} \rightarrow \Delta y$ in $L^{2}(\Omega)$, we obtain (2.16) for $(v, y)$ by passing to the limit in (2.18).

Now, let us introduce the functional

$$
\rho(t)=2 \int_{\Omega} y^{\prime}(m \cdot \nabla y) d x+(n-1) \int_{\Omega} y^{\prime} y d x+C_{0} \int_{\Omega} y^{\prime} \varphi d x
$$

where $\varphi$ is the solution of equation (2.12) and $C_{0}$ is a positive constant to be determined later on $(\sec (2.30))$.

We note that the functional $\rho(t)$ is a slight, but essential modification of the one introduced in Zuazua ([19]), by adding the last integral term. The idea of the proof of the following lemma is to estimate the integral $\int_{\Omega} y^{\prime} \varphi^{\prime} \mathrm{dx}$. We show that with a suitable estimate 
of this term, we can overcome the difficulty caused by the nonlinear feedback and by the amplitude of the function $\alpha$. This point seems to be new.

We have the following important result:

Lemma 2.5 Under the assumptions of Theorem 1, there exist some positive constants $\mathrm{C}_{3}, \mathrm{C}_{4}$ and $\mathrm{C}_{5}$ such that the following estimates hold:

$$
\begin{aligned}
& |\rho(t)| \leq C_{3} E(t) \\
& \rho^{\prime}(t) \leq-E(t)+C_{4} \int_{\Gamma}\left|y^{\prime}\right| 2 d \Gamma+C_{5} \int_{\Gamma} g^{2}\left(y^{\prime}\right) d \Gamma
\end{aligned}
$$

for any solution of equations (1.3)-(1.5).

Proof First of all, it is easy to deduce (2.20) from (2.19) by applying the CauchySchwarz inequality and (2.13). The constant $C_{3}$ is given by

$$
C_{3}=2 R+(n-1) \gamma+\frac{C_{0} \beta}{\sqrt{\alpha_{0}}}
$$

where $\gamma$ is the best constant such that

$$
\int_{\Omega} \psi^{2} \mathrm{dx} \leq \gamma^{2}\left\{\int_{\Omega}|\nabla \psi|^{2} \mathrm{dx}+\int_{\Gamma} \alpha|\psi|^{2} \mathrm{~d} \Gamma\right\}, \forall \psi \in \mathrm{H}^{1}(\Omega) .
$$

Next, we calculate the derivative $\rho^{\prime}(t)$

$$
\begin{aligned}
& \rho^{\prime}(\mathrm{t})=-\mathrm{C}_{0} \int_{\Omega} \nabla \mathrm{y} \nabla \varphi \mathrm{dx}+\mathrm{C}_{0} \int_{\Omega} \mathrm{y}^{\prime} \varphi^{\prime} \mathrm{dx}+2 \int_{\Omega} \Delta \mathrm{y}(\mathrm{m} \cdot \nabla \mathrm{y}) \mathrm{dx}-(\mathrm{n}-1) \int_{\Omega}|\nabla \mathrm{y}| 2 \mathrm{dx} \\
& -\int_{\Omega}\left|\mathrm{y}^{\prime}\right|{ }^{2} \mathrm{dx}+\left(\mathrm{n}-1+\mathrm{C}_{0}\right) \int_{\Gamma} \frac{\partial \mathrm{y}}{\partial v} \mathrm{yd} \Gamma+\int_{\Gamma}(\mathrm{m} \cdot \mathrm{v})\left|\mathrm{y}^{\prime}\right| 2 \mathrm{~d} \Gamma .
\end{aligned}
$$

We deduce from (2.14) that the first term of the right-hand side of (2.24) is negative $\left(C_{0} \geq 0\right)$. For the second term of (2.24), by applying Young's inequality, we deduce from (2.13) that 
(2.25) $\mathrm{C}_{0} \int_{\Omega} \mathrm{y}^{\prime} \varphi^{\prime} \mathrm{dx} \leq \frac{1}{2} \int_{\Omega}\left|\mathrm{y}^{\prime}\right| 2 \mathrm{dx}+\frac{\mathrm{C}_{0}^{2}}{2} \int_{\Omega}\left|\varphi^{\prime}\right| 2 \mathrm{dx} \leq \frac{1}{2} \int_{\Omega}\left|\mathrm{y}^{\prime}\right| 2 \mathrm{dx}+\frac{\left(\mathrm{C}_{0} \beta\right)^{2}}{2} \int_{\Gamma}\left|\mathrm{y}^{\prime}\right| 2 \mathrm{~d} \Gamma$.

In order to apply Lemma 2.4 to estimate the third term of the right-hand side of (2.24), we need to verify that $-\left\{\alpha y+g\left(y^{\prime}\right)\right\} \in L^{2}(\Gamma)$. This is obvious, since $y^{\prime} \in L^{\infty}\left(\mathbb{R}^{+}, H^{1}(\Omega)\right)$ and $|\mathrm{g}(\mathrm{s})| \leq \mathrm{C}\{1+|\mathrm{s}|\}$ (see(1.6) and (2.7)).

Combining (2.16), (2.24) and (2.25), we deduce that

$$
\begin{aligned}
& \rho^{\prime}(t) \leq-\frac{1}{2} \int_{\Omega}\left(|\nabla y|^{2}+\left|y^{\prime}\right|^{2}\right) \mathrm{d} x+\left(\mathrm{R}+\frac{\left(\mathrm{C}_{0} \beta\right)^{2}}{2}\right) \int_{\Gamma}\left|y^{\prime}\right| 2 \mathrm{~d} \Gamma \\
& +\theta \mathrm{R}^{2} \delta \int_{\Gamma}\left|\frac{\partial y}{\partial v}\right| 2 \mathrm{~d} \Gamma+\left(\mathrm{n}-1+\mathrm{C}_{0}\right) \int_{\Gamma} \frac{\partial y}{\partial v} \mathrm{yd} \Gamma
\end{aligned}
$$

where $\theta \geq 1$ is a constant to be determined later on (see(2.29)).

On the other hand, taking into account the boundary condition (1.4), we have

$$
\int_{\Gamma}\left|\frac{\partial \mathrm{y}}{\partial v}\right| 2 \mathrm{~d} \Gamma \leq-\|\alpha\| \int_{\Gamma} \alpha|\mathrm{y}| 2 \mathrm{~d} \Gamma-2\|\alpha\| \int_{\Gamma} \frac{\partial \mathrm{y}}{\partial v} \mathrm{yd} \Gamma+\frac{\|\alpha\|}{\alpha_{0}} \int_{\Gamma} \mathrm{g}^{2}\left(\mathrm{y}^{\prime}\right) \mathrm{d} \Gamma
$$

where we have set $\|\alpha\|=\|\alpha\|_{L^{\infty}(\Gamma)}$.

Plugging (2.27) into (2.26), we obtain

$$
\begin{aligned}
\rho^{\prime}(t) \leq- & \frac{1}{2} \int_{\Omega}\left(|\nabla y| 2+\left|y^{\prime}\right| 2\right) d x-\|\alpha\| \theta R^{2} \delta \int_{\Gamma} \alpha|y| 2 d \Gamma+\theta R^{2} \delta \frac{\|\alpha\|}{\alpha_{0}} \int_{\Gamma} g^{2}\left(y^{\prime}\right) d \Gamma \\
& +\left(R+\frac{\left(C_{0} \beta\right)^{2}}{2}\right) \int_{\Gamma}\left|y^{\prime}\right| 2 d \Gamma+\left(n-1+C_{0}-2 \theta R^{2} \delta\|\alpha\|\right) \int_{\Gamma} \frac{\partial y}{\partial v} y d \Gamma .
\end{aligned}
$$

Finally, with the following choices of constants $\theta$ and $\mathrm{C}_{0}$ :

$$
\begin{aligned}
& \theta=\operatorname{Max}\left\{1, \frac{1}{2 R^{2} \delta\|\alpha\|}, \frac{n-1}{2 R^{2} \delta\|\alpha\|}\right\} \\
& C_{0}=2 \theta R^{2} \delta\|\alpha\|-(n-1) \geq 0
\end{aligned}
$$


we deduce from $(2.28)$ that

$$
\rho^{\prime}(t) \leq-E(t)+C_{4} \int_{\Gamma}\left|y^{\prime}\right| 2 d \Gamma+C_{5} \int_{\Gamma} g^{2}\left(y^{\prime}\right) d \Gamma
$$

where $C_{4}=R+\frac{\left(C_{0} \beta\right)^{2}}{2}$, and $C_{5}=\theta R^{2} \delta \frac{\|\alpha\|}{\alpha_{0}}$

The proof of Lemma 2.5 is now completed.

Remark 2.6 The proof of Lemma 2.5 consists of two essential steps:

(i) Estimation of the term $-\mathrm{C}_{0} \int_{\Omega} \nabla \mathrm{y} \nabla \varphi \mathrm{dx}$.

If, as in the standard procedure, we apply the Cauchy-Schwarz inequality to the integral term $-\mathrm{C}_{0} \int_{\Omega} \nabla \mathrm{y} \nabla \varphi \mathrm{dx}$, we have to control this term by $\mathrm{C}_{0} \int_{\Omega}|\nabla \mathrm{y}|^{2} \mathrm{dx}$. But we find immediately that this estimation requires the smallness of the constant $C_{0}$. This is just what we want to avoid. Fortunately, we can omit the term $-\mathrm{C}_{0} \int_{\Omega} \nabla \mathrm{y} \nabla \varphi \mathrm{dx}$, which is negative by virtue of (2.14). Furthermore, we know that $\int_{\Omega} \nabla \mathrm{y} \nabla \varphi \mathrm{dx} \leq\left(\int_{\Omega \mid}|\nabla \mathrm{y}|^{2} \mathrm{dx}\right)^{1 / 2} \cdot\left(\int_{\Omega}|\nabla \varphi|^{2} \mathrm{dx}\right)^{1 / 2} \leq \int_{\Omega}|\nabla \mathrm{y}|^{2} \mathrm{dx}$, because $\varphi$ is the function which has the least norm and takes the same values as $y$ on the boundary $\Gamma$ ( see Remark 2.3 ).

(ii) Estimation of the term $C_{0} \int_{\Omega} y^{\prime} \varphi^{\prime} d x$.

Using Young's inequality, this term can be dominated by the sum of the two terms $\frac{1}{2} \int_{\Omega}|y|^{2} \mathrm{dx}+\frac{\mathrm{C}_{0}^{2}}{2} \int_{\Omega}|\varphi|^{2} \mathrm{dx}$. The first one with a suitable coefficient (i.e. 1/2) can be easily handled. Therefore, we have to affect to the second one an arbitrary coefficient (i.e. $C^{2} / 2$ ). By means of (2.13), we can transform $\int_{\Omega}\left|\varphi^{\prime}\right| 2 \mathrm{dx}$ into a integral term on the boundary $\int_{\Gamma}\left|\varphi^{\prime}\right|^{2} \mathrm{~d} \Gamma$. In other words, for estimating the term $\int_{\Omega} y^{\prime} \varphi^{\prime} \mathrm{dx}$, we distinguish the roles of the functions $y^{\prime}$ and $\varphi^{\prime}$, and we evaluate $\int_{\Omega}\left|\varphi^{\prime}\right|^{2} \mathrm{dx}$ by $\int_{\Gamma}\left|\varphi^{\prime}\right|^{2} \mathrm{~d} \Gamma$. If we replace the function $\varphi$ by $y$ in (2.19), instead of $-\mathrm{C}_{0} \int_{\Omega} \nabla \mathrm{y} \nabla \varphi \mathrm{dx}$, we obtain $-\mathrm{C}_{0} \int_{\Omega}|\nabla \mathrm{y}|^{2} \mathrm{dx}$ which is also negative. There is no problem in step (i). However, in place of $C_{0} \int_{\Omega} y^{\prime} \varphi^{\prime} d x$, we have to consider $C_{0} \int_{\Omega}\left|y^{\prime}\right| 2 d x$. We meet again the troublesome problem of the smallness of $\mathrm{C}_{0}$. 
Now, we see clearly why we have been able to control the term $\int_{\Omega} y^{\prime} \varphi^{\prime} d x$ : we have chosen the function $\varphi$ as the second multiplier which allows us to evaluate the domain integral $\int_{\Omega}\left|\varphi^{\prime}\right| 2 \mathrm{dx}$ by the boundary integral $\int_{\Gamma}\left|\mathrm{y}^{\prime}\right| 2 \mathrm{~d} \Gamma$.

Remark 2.7 We have introduced the constant $\theta \geq 1$ for a technical reason. In fact, if we take $\theta=1$, then for any $\alpha$ such that $\|\alpha\| \geq(n-1) / 2 \delta R^{2}$, we have $C_{0} \geq 0$. Thus Lemma 2.5 is true. In the opposite case: $0<\|\alpha\|<(n-1) / 2 \delta R^{2}$, Theorem 1 has been proved by Zuazua in [19].

\section{Proof of Theorem 1}

From now on, the method which we will adopt is analogous to the one used in [19]. Let us recall some essential steps for the sake of completeness.

First, by the conditions (1.10) and (1.12), we deduce from (2.21) that

$$
\rho^{\prime}(t) \leq-E(t)+C_{6} \int_{\Gamma}\left|y^{\prime}\right| 2 d \Gamma
$$

with the constant $\mathrm{C}_{6}=\mathrm{C}_{4}+\mathrm{C}_{2}^{2} \mathrm{C}_{5}$.

Next, for $\varepsilon>0$, we introduce a perturbed energy $E_{\varepsilon}$

$$
E_{\varepsilon}(t)=E(t)+\varepsilon(E(t))^{\frac{p-1}{2}} \rho(t)
$$

Since the energy is nonincreasing, given any $M>1$, we show easily that the following inequalities hold

$$
M^{\frac{-1}{2}}\left(E_{\varepsilon}(t)\right)^{\frac{p+1}{2}} \leq(E(t))^{\frac{p+1}{p}} \leq M^{\frac{1}{2}}\left(E_{\varepsilon}(t)\right)^{\frac{p+1}{2}}
$$

provided $\varepsilon$ is chosen such that

$$
\varepsilon \leq C_{3}^{-1}(E(0))^{\frac{1-p}{2}}\left(1-M^{-\frac{1}{p+1}}\right)=C_{7}(E(0))^{\frac{1-p}{2}}
$$

Now, we calculate the derivative of the perturbed energy $E_{\varepsilon}(t)$ 


$$
E_{\varepsilon}^{\prime}(t)=E^{\prime}(t)+\varepsilon \frac{p-1}{2}(E(t))^{\frac{p-3}{2}} E^{\prime}(t) \rho(t)+\varepsilon(E(t))^{\frac{p-1}{2}} \rho^{\prime}(t)
$$

Combining (1.9), (2.20), (2.31) and (2.35), we get:

(2.36) $\mathrm{E}_{\varepsilon}^{\prime}(\mathrm{t}) \leq\left(-1+\varepsilon \frac{\mathrm{p}-1}{2} \mathrm{C}_{3}(\mathrm{E}(0))^{\frac{\mathrm{p}-1}{2}}\right) \int_{\Gamma} \mathrm{g}\left(\mathrm{y}^{\prime}\right) \mathrm{y}^{\prime} \mathrm{d} \Gamma+\varepsilon \mathrm{C}_{6}(\mathrm{E}(\mathrm{t}))^{\frac{\mathrm{p}-1}{2}} \int_{\Gamma}\left|\mathrm{y}^{\prime}\right| 2 \mathrm{~d} \Gamma-\varepsilon(\mathrm{E}(\mathrm{t}))^{\frac{\mathrm{p}+1}{2}}$.

On the other hand, by (1.10) and (1.12), we deduce that

$$
\varepsilon C_{6}(E(t))^{\frac{p-1}{2}}\left\{\int_{\mid y^{\prime}}\left|y_{\geq 1}\right| 2 d \Gamma \leq\left(1-\varepsilon \frac{p-1}{2} C_{3}(E(0))^{\frac{p-1}{2}}\right)\left\{\int_{\left\{y^{\prime} \mid \geq 1\right\}}^{g}\left(y^{\prime}\right) y^{\prime} d \Gamma\right.\right.
$$

if we choose $\varepsilon>0$ such that

$$
\varepsilon \leq C_{1}(E(0))^{\frac{1-p}{2}}\left(C_{1} C_{3} \frac{p-1}{2}+C_{6}\right)^{-1}=C_{8}(E(0))^{\frac{1-p}{2}}
$$

From (2.36) and (2.37), it follows:

$$
\begin{aligned}
& E_{\varepsilon}^{\prime(t) \leq}\left(-1+\varepsilon \frac{p-1}{2} C_{3}(E(0))^{\frac{p-1}{2}}\right)\left\{\int_{\left\{\left|y^{\prime}\right| \leq 1\right\}}^{g\left(y^{\prime}\right) y^{\prime} d \Gamma+}\right. \\
& \varepsilon C_{6}(E(t))^{\frac{p-1}{2}}\left\{\left.\int_{\left\{\mid y^{\prime}\right.}^{\left.\left|y^{\prime}\right| 2 d\right\}}\right|^{\frac{p+1}{2}}-\varepsilon(E(t))^{\frac{1}{2}} .\right.
\end{aligned}
$$

Now we distinguish the cases $\mathrm{p}=1$ and $\mathrm{p}>1$.

(i) case $p=1$. In this case (1.10), (2.33), (2.38) and (2.39) yield:

$$
E_{\varepsilon}^{\prime}(t) \leq-\varepsilon E(t) \leq-\varepsilon M^{\frac{-1}{2}} E_{\varepsilon}(t),
$$

consequently, we get:

$$
\mathrm{E}_{\varepsilon}(\mathrm{t}) \leq \mathrm{E}_{\varepsilon}(0) \mathrm{e}^{-\varepsilon \mathrm{M}^{\frac{-1}{2}} \mathrm{t}}, \quad \forall \mathrm{t} \geq 0,
$$


which, together with (2.33), implies:

$$
\mathrm{E}(\mathrm{t}) \leq \mathrm{ME}(0) \mathrm{e}^{-\varepsilon \mathrm{M}^{\frac{-1}{2}} \mathrm{t}}=\mathrm{ME}(0) \mathrm{e}^{-\lambda \mathrm{t}}, \quad \forall \mathrm{t} \geq 0 \text {, }
$$

where we have set $\lambda=\varepsilon M^{-\frac{1}{2}}$.

From (2.34) and (2.38), we observe that the constant $\varepsilon$ does not depend on $E(0)$, hence the constant $\lambda$ defined above does not depend on $\mathrm{E}(0)$ too. This will not be the case when p $>1$ (see Remark 2.9 ).

(ii) case $p>1$. From (2.39) and by Young's inequality, we have:

$$
\begin{aligned}
& E_{\varepsilon}^{\prime}(t) \leq\left(-1+\varepsilon \frac{p^{-1}}{2} C_{3}(E(0))^{\frac{p-1}{2}}\right) \quad\left\{\int_{\left\{\left|y^{\prime}\right| \leq 1\right\}}^{g}\left(y^{\prime}\right) y^{\prime} d \Gamma+\right. \\
& \varepsilon\left(2 C_{6}\right)^{\frac{p-1}{2}}\left(C_{\left\{\mid y^{\prime}\right.}\left\{\left.\right|_{\leq 1\}} \mid 2 d \Gamma\right)^{\frac{p+1}{2}}-\frac{\varepsilon}{2}(E(t))^{\frac{p+1}{2}} .\right.
\end{aligned}
$$

We apply Hölder's inequality with the exponents $\alpha=\frac{p+1}{p-1}, \alpha^{\prime}=\frac{p+1}{2}$ to the second term of the right-hand side of (2.42), it follows:

$$
\left(\int_{\left\{\left|y^{\prime}\right| \leq 1\right\}}\left|y^{\prime}\right| 2 d \Gamma\right)^{\frac{p+1}{2}} \leq\left(\int_{\left\{\left|y^{\prime}\right| \leq 1\right\}} d \Gamma\right)^{\frac{p-1}{2}} \cdot \iint_{\left\{\left|y^{\prime}\right| \leq 1\right\}}\left|y^{\prime}\right| p+1 d \Gamma \leq(\operatorname{meas} \Gamma)^{\frac{p-1}{2}} \cdot \int_{\left\{\left|y^{\prime}\right| \leq 1\right\}}\left|y^{\prime}\right| p+1 d \Gamma .
$$

The above estimate, together with (1.12) and (2.42), implies the following inequality:

$$
\begin{aligned}
& E_{\varepsilon}^{\prime}(t) \leq\left(-1+\varepsilon \frac{p^{p}-1}{2} C_{3}(E(0))^{\frac{p-1}{2}}\right) \int_{\left\{\left|y^{\prime}\right| \leq 1\right\}}^{g}\left(y^{\prime}\right) y^{\prime} d \Gamma+ \\
& \varepsilon C_{1}^{-1}\left(2 C_{6}\right)^{\frac{p-1}{2}}(\text { meas } \Gamma)^{\frac{p-1}{2}}\left\{\int_{\left\{\mid y^{\prime}\{\leq 1\}\right.}^{g\left(y^{\prime}\right) y^{\prime} d \Gamma-\frac{\varepsilon}{2}(E(t))^{\frac{p+1}{2}} .}\right.
\end{aligned}
$$

We have thus proved 


$$
\left.\mathrm{E}_{\varepsilon}^{\prime}(\mathrm{t}) \leq-\frac{\varepsilon}{2} \mathrm{E}^{\frac{\mathrm{p}+1}{2}}\right)^{2}, \quad \forall \mathrm{t} \geq 0
$$

provided $\varepsilon$ satisfies:

$$
\begin{aligned}
& \varepsilon \leq C_{1}\left(C_{1} C_{2} \frac{p-1}{2}(E(0))^{\frac{\mathrm{p}-1}{2}}+\left(2 C_{6}\right)^{\frac{\mathrm{p}-1}{2}}(\text { meas } \Gamma)^{\frac{\mathrm{p}-1}{2}}\right)^{-1}= \\
& (E(0))^{\frac{1-\mathrm{p}}{2}}\left(\mathrm{C}_{9}+\mathrm{C}_{10}(\mathrm{E}(0))^{\frac{1-\mathrm{p}}{2}}\right)^{-1} .
\end{aligned}
$$

Combining (2.33) and (2.43), we get:

$$
E_{\varepsilon}^{\prime}(t) \leq-\frac{\varepsilon}{2} M^{-\frac{1}{2}}\left(E_{\varepsilon}(t)\right)^{\frac{p+1}{2}}
$$

Finally, solving the differential inequality (2.45) and using (2.33), we obtain

$$
\mathrm{E}(\mathrm{t}) \leq \mathrm{ME}(0)\left\{1+\mathrm{t} \frac{\varepsilon}{4} \frac{\mathrm{p}-1}{\mathrm{p}} \mathrm{M}^{\frac{-\mathrm{p}}{\mathrm{p}+1}}(\mathrm{E}(0))^{\frac{\mathrm{p}-1}{2}}\right\}^{\frac{-2}{\mathrm{p}-1}}=\mathrm{ME}(0)\{1+\mu \mathrm{t}\}^{\frac{-2}{\mathrm{p}-1}}
$$

The proof of Theorem 1 is thus completed.

Remark 2.9 The constant $\varepsilon$ is chosen such that the restrictions (2.34) (2.38) and (2.44) are satisfied. In fact, we can choose $\varepsilon$ as follows:

$$
\varepsilon=\operatorname{Min}\left(C_{7}, C_{8},\left(C_{9}+C_{10}(E(0))^{\frac{1-p}{2}}\right)^{-1}\right)(E(0))^{\frac{1-p}{2}}
$$

with constants $\mathrm{C}_{7}, \mathrm{C}_{8}, \mathrm{C}_{9}$ and $\mathrm{C}_{10}$ independent of $\mathrm{E}(0)$. In particular, we conclude that $\varepsilon$ and consequently $\mu=\frac{\varepsilon(p-1)}{4} M-\frac{p}{p+1}(E(0)) \frac{p-1}{2}$, depend continuously on $E(0)$.

\section{Proof of Theorem 2}

First, by the conditions (1.14) and (2.21), we have

$$
\rho^{\prime}(t) \leq-E(t)+C_{6} \int g^{2}\left(y^{\prime}\right) d \Gamma
$$


with the constant $\mathrm{C}_{6}=\mathrm{C}_{5}+\frac{\mathrm{C}_{4}}{\mathrm{C}_{1}^{2}}$.

Next, for $\varepsilon>0$, we introduce the perturbed energy $E_{\varepsilon}$

$$
E_{\varepsilon}(t)=E(t)+\varepsilon(E(t))^{\frac{1-p}{2 p}} \rho(t)
$$

Then, for any $M>1$, we have

$$
M^{\frac{-1}{2}}\left(E_{\varepsilon}(t)\right)^{\frac{p+1}{2 p}} \leq(E(t))^{\frac{p+1}{2 p}} \leq M^{\frac{1}{2}}\left(E_{\varepsilon}(t)\right)^{\frac{p+1}{2 p}}
$$

provided $\varepsilon$ satisfies

$$
\varepsilon \leq C_{3}^{-1}(E(0))^{\frac{p-1}{2 p}}\left(1-M^{\frac{p-1}{2 p}}\right)=C_{7}(E(0))^{\frac{p-1}{2 p}} .
$$

We have also

$$
E_{\varepsilon}^{\prime}(t)=E^{\prime}(t)+\varepsilon \frac{1-p}{2 p}(E(t))^{\frac{1-3 p}{2 p}} E^{\prime}(t) \rho(t)+\varepsilon(E(t))^{\frac{1-p}{2 p}} \rho^{\prime}(t) .
$$

Combining (1.9), (2.20), (2.48), and (2.52), we get

$$
\begin{aligned}
& E_{\varepsilon}(t) \leq\left\{-1+\varepsilon \frac{1-p}{2 p} C_{3}(E(0))^{\frac{1-p}{2 p}}\right\} \int_{\Gamma} g\left(y^{\prime}\right) y^{\prime} d \Gamma+ \\
& \varepsilon C_{6}(E(t))^{\frac{1-p}{2 p}} \int_{\Gamma} g^{2}\left(y^{\prime}\right) d \Gamma-\varepsilon(E(t))^{\frac{1+p}{2 p}} .
\end{aligned}
$$

By the conditions (1.14) we have

$$
\left.\lg (s)\right|^{2} \leq \mathrm{C}_{2} \mathrm{~g}(\mathrm{~s}) \mathrm{s}, \quad \text { for }|\mathrm{s}| \geq 1
$$

$$
|g(s)|^{2} \leq\left(C_{2}\right)^{\frac{2}{p+1}}(g(s) s)^{\frac{2 p}{p+1}}, \quad \text { for }|s|<1
$$

We deduce from (2.54)

$$
\varepsilon C_{6}(E(t))^{\frac{1-p}{2 p}}\left\{\int_{\mid y^{\prime}} g_{\geq 1\}}^{2}\left(y^{\prime}\right) d \Gamma \leq\left\{1-\varepsilon \frac{1-p}{2 p} C_{3}(E(0))^{\frac{1-p}{2 p}}\right\} \quad\left\{\int_{y^{\prime}} g\left(y^{\prime}\right) y^{\prime} d \Gamma\right.\right.
$$


provided $\varepsilon$ is chosen such that

$$
\varepsilon \leq(E(0))^{\frac{p-1}{2 p}}\left\{C_{2} C_{6}+\frac{1-p}{2 p} C_{3}\right\}^{-1}=C_{8}(E(0))^{\frac{p-1}{2 p}}
$$

From (2.53) and (2.56), it follows

$$
\begin{aligned}
& E_{\varepsilon}(t) \leq\left\{-1+\varepsilon \frac{1-p}{2 p} C_{3}(E(0))^{\frac{1-p}{2 p}}\right\} \quad\left\{\left.\int_{y^{\prime}}\right|_{\leq 1\}}\left(y^{\prime}\right) y^{\prime} d \Gamma+\right. \\
& \left.\varepsilon C_{6}(E(t))^{\frac{1-p}{2 p}}\left(\left|y^{\prime}\right| \leq 1\right\}\right\} \\
& g^{2}\left(y^{\prime}\right) d \Gamma-\varepsilon(E(t))^{\frac{1+p}{2 p}} .
\end{aligned}
$$

From (2.55) and by Young's inequality, we have

$$
\begin{aligned}
& \varepsilon C_{6}(E(t))^{\frac{1-p}{2 p}}\left(\mid y_{y^{\prime}} \int_{\leq 1\}} g^{2}\left(y^{\prime}\right) d \Gamma \leq \frac{\varepsilon}{2}(E(t))^{\frac{1+p}{2 p}}+\varepsilon\left(2 C_{6}\right)^{\frac{1+p}{2 p}}\left(\int_{\left(\left|y^{\prime}\right| \leq 1\right\}} g^{2}\left(y^{\prime}\right) d \Gamma\right)^{\frac{1+p}{2 p}}\right. \\
& \leq \frac{\varepsilon}{2}(E(t))^{\frac{1+p}{2 p}}+\varepsilon\left(2 C_{6}\right)^{\frac{1+p}{2 p}}\left(C_{2}\right)^{\frac{1}{p}}\left\{\int_{\left|y^{\prime}\right|_{\leq 1\}}}\left(g\left(y^{\prime}\right) y^{\prime}\right)^{\frac{2 p}{1+p}} d \Gamma\right\}^{\frac{1+p}{2 p}} .
\end{aligned}
$$

We apply Hölder's inequality with exponents $\alpha=\frac{p+1}{2 p}, \alpha^{\prime}=\frac{p+1}{1-p}$ to the last term of the righthand side of (2.59), to obtain

$$
\begin{aligned}
& \left\{\int_{\left\{\left|y^{\prime}\right| \leq 1\right\}}\left(g\left(y^{\prime}\right) y^{\prime}\right)^{\frac{2 p}{1+p}} d \Gamma\right\}^{\frac{1+p}{2 p}} \leq\left\{\int_{\left\{y^{\prime} \mid \leq 1\right\}} g\left(y^{\prime}\right) y^{\prime} d \Gamma \cdot\left\{\int_{\left\{y^{\prime} \mid \leq 1\right\}} d \Gamma\right\}^{\frac{1-p}{2 p}}\right. \\
& \leq\left\{y^{\prime}\{\leq 1\}\right. \\
& g\left(y^{\prime}\right) y^{\prime} d \Gamma \cdot\{\operatorname{meas} \Gamma\}^{\frac{1-p}{2 p}} .
\end{aligned}
$$

Inserting (2.59), (2.60) into (2.58) yields

$$
E_{\varepsilon}^{\prime}(t) \leq\left\{-1+\varepsilon \frac{1-p}{2 p} C_{3}(E(0))^{\frac{1-p}{2 p}}\right\} \quad\left\{\int_{y^{\prime}} g\left(y_{\leq 1}\right) y^{\prime} d \Gamma\right.
$$




$$
+\varepsilon\left(2 C _ { 6 } ^ { \frac { 1 + p } { 2 p } } ( C _ { 2 } ) ^ { \frac { 1 } { p } } ( \text { meas } \Gamma ) ^ { \frac { 1 - p } { 2 p } } \left\{\int_{\left.\left|y^{\prime}\right| \leq 1\right\}}^{g}\left(y^{\prime}\right) y^{\prime} d \Gamma-\frac{\varepsilon}{2}(E(t))^{\frac{1+p}{2 p}} .\right.\right.
$$

By choosing $\varepsilon$ so that

$$
\varepsilon \leq\left\{\frac{1-p^{2}}{2 p} C_{3}(E(0))^{\frac{1-p}{2 p}}+\left(2 C_{6}\right)^{\frac{1+p}{2 p}}\left(C_{2}\right)^{\frac{1}{p}}(\text { meas } \Gamma)^{\frac{1-p}{2 p}}\right\}^{-1}
$$

we deduce from (2.61) that

$$
E_{\varepsilon}^{\prime}(t) \leq-\frac{\varepsilon}{2}(E(t))^{\frac{1+p}{2 p}}
$$

Finally, solving the differential inequality (2.63), using (2.50), we obtain

$$
E(t) \leq M E(0)\left\{1+t \frac{\varepsilon}{4} \frac{1-p}{p} M^{\frac{-1}{p+1}}(E(0))^{\frac{1-p}{2 p}}\right\}^{\frac{-2 p}{1-p}}=M E(0)\{1+\mu t\}^{\frac{-2 p}{1-p}}
$$

The proof of Theorem 2 is thus completed.

Remark 2.10. One can also consider the problem where the boundary condition

$$
\frac{\partial y}{\partial v}+\alpha y=-g\left(y^{\prime}\right) \quad \text { on } \Gamma
$$

is replaced by the conditions

$$
\begin{aligned}
& \frac{\partial y}{\partial v}+\alpha y=-g\left(y^{\prime}\right) \quad \text { on } \Gamma_{0}=\{x \in \Gamma ; m . v>0\} \\
& y=0 \quad \text { on } \Gamma_{*}=\Gamma \backslash \Gamma_{0},
\end{aligned}
$$

as in [19]. Then, with the assumption (1.2) on $\Gamma_{0}$, which implies $\bar{\Gamma}_{0} \cap \bar{\Gamma}_{*}=\varnothing$, we have the same results as before, for any $\alpha \geq \alpha_{0}>0$. In [19], the assumption (1.2) is not necessary (and in fact not true), but $\alpha$ has to be small. Thus for this variant, the results of [19] are not the same as ours.

However, in the case of Dirichlet boundary conditions on part of the boundary, the results are true without any $\alpha[19]$. The interest in considering an $\alpha$ in that case is in the gain of robustness, as shown by Zuazua [20]. 


\section{$\$ 3$ Wave equation with a variable coefficient}

In arbitrary space dimension, some recent results about the stabilization problem for the wave equation with variable coefficients can be found in [9], [10]. There the author considers regular coefficients satisfying a specific coercivity condition.

In this section, taking a more general multiplier than $(\mathrm{m} . \nabla \mathrm{y})$, we show that the results of the previous section are still valid in the one dimensional case for a large class of coefficients. However the extension of this method to the case of arbitrary space dimension seems difficult.

Let us consider the following equation:

$$
\begin{aligned}
& y^{\prime \prime}-\left(\operatorname{ay}_{x}\right)_{x}=0 \quad \text { in }(0,1) \times(0 \infty), \\
& \begin{cases}\operatorname{ay}_{x}(0, t)-\alpha y(0, t)=g\left(y^{\prime}(0, t)\right) & t>0, \\
\operatorname{ay}_{x}(1, t)+\alpha y(1, t)=-g\left(y^{\prime}(1, t)\right) & t>0,\end{cases} \\
& y(0)=y_{0} \in H^{1}(0,1), y^{\prime}(0)=y_{1} \in L^{2}(0,1)
\end{aligned}
$$

where $\alpha$ is a positive constant, and $\mathrm{g}: \mathbb{R} \longrightarrow \mathbb{R}$ is a continuous nondecreasing function such that

$$
g(0)=0, \quad g(s) s>0, \quad \text { for all } s \neq 0
$$

We assume that the function a belongs to the space $\mathrm{H}^{1}(0,1)$ and that

$$
0<\underline{\mathrm{a}} \leq \mathrm{a}(\mathrm{x}), \quad 0 \leq \mathrm{x} \leq 1 .
$$

Under the assumptions (3.4) and (3.5), it is easy to see that the equations (3.1)-(3.3) have a unique strong solution y such that

$$
y \in W^{1, \infty}\left(\mathbb{R}^{+}, H^{1}(0,1)\right) \cap L^{\infty}\left(\mathbb{R}^{+}, H^{2}(0,1)\right)
$$

for every initial data $\left(\mathrm{y}_{0}, \mathrm{y}_{1}\right) \in W$, where $W$ denotes the subset of $H^{1}(0,1) \times L^{2}(0,1)$

$$
\begin{array}{r}
W=\left\{(y, \hat{y}) \in H^{2}(0,1) \times H^{1}(0,1) ;\left(\text { ay }_{x}\right)(0)-\alpha y(0)=g(\hat{y}(0)),\right. \\
\left.\left(\text { ay }_{x}\right)(1)+\alpha y(1)=-g(\hat{y}(1))\right\} .
\end{array}
$$


Furthermore, we can extend the mapping $\left(y_{0}, y_{1}\right) \longrightarrow\left(y(t), y^{\prime}(t)\right)$ to a strongly continuous semi-group of contractions $(S(t))_{\geq \geq 0}$ in $H^{1}(0,1) \times L^{2}(0,1)$. Thus for every initial data $\left(y_{0}, y_{1}\right) \in H^{1}(0,1) \times L^{2}(0,1)$ there exists a unique weak solution of the equations (3.1)(3.3) which is given by the following formula:

$$
\left(y(t), y^{\prime}(t)\right)=S(t)\left(y_{0}, y_{1}\right), \forall t \geq 0
$$

with the regularity

$$
y \in C^{0}\left(\mathbb{R}^{+}, H^{1}(0,1)\right) \cap C^{1}\left(\mathbb{R}^{+}, L^{2}(0,1)\right)
$$

As in the previous section, we assume the regularity (3.6), since the subset $\mathrm{W}$ is dense in the space $\mathrm{H}^{1}(0,1) \times \mathrm{L}^{2}(0,1)$ and $(\mathrm{S}(\mathrm{t}))_{1 \geq 0}$ is a strongly continuous semi-group of contractions.

The strong asymptotic stabilization can be proved by a standard argument (see [1], [5], [17]). As in the previous section, we consider here the problem of estimating the rate of decay.

For any solution $y$ of the equations (3.1)-(3.3), we define the associated energy

$$
E(t)=\frac{1}{2} \int_{0}^{1}\left(a\left|y_{x}\right|^{2}+\left|y^{\prime}\right|^{2}\right) d x+\frac{\alpha}{2}\left(y^{2}(0, t)+y^{2}(1, t)\right)
$$

Theorem 3. Let $g$ be a continuous nondecreasing function such that (3.4) holds. Assume that $a \in H^{1}(0,1)$ satisfies (3.5). Then we have:

(i) If there exist some positive constants $C_{1}, C_{2}$ such that

$$
\mathrm{C}_{1}|\mathrm{~s}| \leq \lg (\mathrm{s})\left|\leq \mathrm{C}_{2}\right| \mathrm{s} \mid, \quad \forall \mathrm{s} \in \mathbb{R},
$$

then given any $M>1$, there exists a constant $\lambda>0$ such that

$$
\mathrm{E}(\mathrm{t}) \leq \mathrm{ME}(0) \mathrm{e}^{-\lambda \mathrm{t}}, \quad \forall \mathrm{t} \geq 0
$$

(ii) If there exist some positive constants $C_{1}, C_{2}$ and $p>1$ such that

$$
\mathrm{C}_{1} \operatorname{Min}(|\mathrm{s}|,|\mathrm{s}| \mathrm{P}) \leq \lg (\mathrm{s})\left|\leq \mathrm{C}_{2}\right| \mathrm{s} \mid, \quad \forall s \in \mathbb{R},
$$

then given any $M>1$, there exists a constant $\mu>0$ depending on $E(0)$ such that

$$
\mathrm{E}(\mathrm{t}) \leq \mathrm{ME}(0)(1+\mu \mathrm{t})^{-\frac{2}{\mathrm{p}-1}}, \quad \forall \mathrm{t} \geq 0 .
$$


Sketch of the proof

First, we replace the functional $\rho(t)$, introduced in the previous section, by the following functional

$$
\rho_{1}(t)=2 \int_{0}^{1} y^{\prime} \varphi y_{x} d x+C_{0} \int_{0}^{1} y^{\prime} \psi d x
$$

where $C_{0}$ is a positive constant and $\psi$ is the solution of the equations

$$
\begin{aligned}
& \left(a \psi_{x}\right)_{x}=0, \\
& \begin{cases}\psi(0, t)=y(0, t) \\
\psi(1, t)=y(1, t)\end{cases}
\end{aligned}
$$

Solving the equations (3.16)-(3.17), we have:

$$
\psi(x, t)=\frac{\int_{0}^{x} a^{-1}(s) d s}{\int_{0}^{1} a^{-1}(s) d s}(y(1, t)-y(0, t))+y(0, t)
$$

We verify that the following inequalities hold

$$
\begin{gathered}
\int_{0}^{1} a \psi_{x} y_{x} d x=\left(\int_{0}^{1} a^{-1}(s) d s\right)^{-1}(y(1, t)-y(0, t))^{2} \geq 0, \\
\int_{0}^{1} \psi^{2} d x \leq 5\left(y^{2}(0, t)+y^{2}(1, t)\right) .
\end{gathered}
$$

The function $\varphi$ is chosen so that 


$$
\varphi \in \mathrm{H}^{1}(0,1), \quad \varphi_{x} \geq 1, \quad \mathrm{a}\left(\frac{\varphi}{\mathrm{a}}\right)_{\mathrm{x}} \geq 1
$$

$$
\varphi(0)<0, \quad \varphi(1)>0,
$$

An example of such a function will be given explicitly ( see (3.36) ).

Next, it is easy to verify that

$$
\left|\rho_{1}(t)\right| \leq C_{3} E(t), \quad \forall t \geq 0,
$$

with

$$
C_{3}=\left\|\frac{\varphi}{\sqrt{a}}\right\|^{\infty}(0,1)+C_{0} \sqrt{\frac{5}{\alpha}}
$$

We calculate the derivative of the functional $\rho_{1}(t)$

$\rho_{1}^{\prime}(t)=\left[a y_{x}^{2} \varphi\right]_{0}^{1}+\left[\left(y^{\prime}\right)^{2} \varphi\right]_{0}^{1}-\int_{0}^{1}\left(a y_{x}^{2}\left(\frac{\varphi}{a}\right)_{x} a+\left|y^{\prime}\right|^{2} \varphi_{x}\right) d x+C_{0}\left[a y_{x} \psi\right]_{0}^{1}-C_{0} \int_{0}^{1} a y_{x} \psi_{x} d x+C_{0} \int_{0}^{1} y^{\prime} \psi^{\prime} d x$

Taking into account (3.18), (3.19), (3.21) and (3.22), we obtain

$$
\begin{aligned}
\rho_{1}^{\prime}(t) \leq & \theta\left[a y_{x}^{2} \varphi\right]_{0}^{1}+\left[\left|y^{\prime}\right|^{2} \varphi\right]_{0}^{1}-\int_{0}^{1}\left(a y_{x}^{2}+\left|y^{\prime}\right|^{2}\right) d x \\
& +C_{0}\left[a y_{x} y\right]_{0}^{1}+C_{0}\left(\int_{0}^{1}\left|y^{\prime}\right|^{2} d x\right)^{\frac{1}{2}}\left(\int_{0}^{1}\left|\psi^{\prime}\right|^{2} d x\right)^{\frac{1}{2}}
\end{aligned}
$$

where $\theta$ is an arbitrary constant satisfying

$$
\theta \geq 1 \text {. }
$$

We apply Young's inequality to the last term of the right-hand side of (3.25), using (3.20) 


$$
\begin{aligned}
& C_{0}\left(\int_{0}^{1}\left|y^{\prime}\right|^{2} d x\right)^{\frac{1}{2}}\left(\int_{0}^{1}\left|\psi^{\prime}\right|^{2} d x\right)^{\frac{1}{2}} \leq \frac{1}{2} \int_{0}^{1}\left|y^{\prime}\right|^{2} d x+\frac{C_{0}^{2}}{2} \int_{0}^{1}\left|\psi^{\prime}\right|^{2} d x \\
& \leq \frac{1}{2} \int_{0}^{1}\left|y^{\prime}\right|^{2} d x+\frac{5 C_{0}^{2}}{2}\left(\left|y^{\prime}(0, t)\right|^{2}+\left|y^{\prime}(1, t)\right|^{2}\right)
\end{aligned}
$$

Inserting (3.27) into (3.25) yields

$$
\begin{aligned}
\left.\rho_{1}^{\prime}(t) \leq \theta\left[a y_{x}^{2} \varphi\right]_{0}^{1}+\left[\left|y^{\prime}\right|^{2} \varphi\right]_{0}^{1}-\frac{1}{2} \int_{0}^{1}\left(a y_{x}^{2}+\left|y^{\prime}\right|^{2}\right) d x\right)+C_{0}\left[a y_{x} y\right]_{0}^{1} \\
+\frac{5 C_{0}^{2}}{2}\left(\left|y^{\prime}(0, t)\right|^{2}+\left|y^{\prime}(1, t)\right|^{2}\right)
\end{aligned}
$$

On the other hand, using the boundary conditions (3.2), a straightforward computation shows that

$$
\left[\operatorname{ay}_{\mathrm{x}}^{2} \varphi\right]_{0}^{1}=-\alpha^{2}\left[\frac{\varphi}{\mathrm{a}} \mathrm{y}^{2}\right]_{0}^{1}+\left[\frac{\mathrm{g}^{2}\left(\mathrm{y}^{\prime}\right)}{\mathrm{a}} \varphi\right]_{0}^{1}-2 \alpha\left(\varphi(0) \mathrm{y}_{\mathrm{x}}(0, \mathrm{t}) \mathrm{y}(0, \mathrm{t})+\varphi(1) \mathrm{y}_{\mathrm{x}}(1, \mathrm{t}) \mathrm{y}(1, \mathrm{t})\right)
$$

Eliminating the term $\left[\operatorname{ay}_{x}^{2} \varphi\right]_{0}^{1}$ appearing in (3.28) and (3.29), using (3.22) and (3.23), we get

$$
\begin{aligned}
\rho_{1}^{\prime}(t) \leq & -\frac{1}{2} \int_{0}^{1}\left(a y_{x}^{2}+\left|y^{\prime}\right|^{2}\right) d x-\theta \alpha^{2} C_{4}\left(|y(0, t)|^{2}+|y(1, t)|^{2}\right) \\
& +\left(C_{5}+\frac{5 C_{0}^{2}}{2}\right)\left(\left|y^{\prime}(0, t)\right|^{2}+\left|y^{\prime}(1, t)\right|^{2}\right)+C_{5} \theta\left(\frac{\mid g^{2}\left(y^{\prime}(0, t)\right)}{a(0)}+\frac{g^{2}\left(y^{\prime}(1, t)\right)}{a(1)}\right) \\
& +\left(C_{0}-2 \alpha \theta \frac{\varphi(1)}{a(1)}\right){ }_{\left[a y_{x} y\right]_{0}^{1}}
\end{aligned}
$$

where we have set 


$$
\left\{\begin{array}{l}
C_{4}=\operatorname{Min}\left\lfloor-\frac{\varphi(0)}{a(0)}, \frac{\varphi(1)}{a(1)}\right\} \\
C_{5}=\operatorname{Max}(-\varphi(0), \varphi(1))
\end{array}\right.
$$

Now, if we choose the constants $\theta$ and $\mathrm{C}_{0}$ as follows

$$
\theta=\operatorname{Max}\left\{1, \frac{1}{2 \alpha C_{4}}\right)
$$

$$
C_{0}=2 \alpha \theta \frac{\varphi(1)}{a(1)}>0
$$

we deduce from (3.30) that

$$
\rho_{1}^{\prime}(t) \leq-E(t)+C_{6}\left(\left|y^{\prime}(0, t)\right|^{2}+\left|y^{\prime}(1, t)\right|^{2}\right)+C_{7}\left(g^{2}\left(y^{\prime}(0, t)+g^{2}\left(y^{\prime}(1, t)\right)\right)\right.
$$

with the constants $C_{6}$ and $C_{7}$ given by

$$
\left\{\begin{array}{l}
C_{6}=C_{5}+\frac{5 C_{0}^{2}}{2}, \\
C_{7}=\operatorname{Max}\left(\frac{1}{a(0)}, \frac{1}{a(1)}\right) C_{5} \theta
\end{array}\right.
$$

We see that the estimate (3.34) will play the same role than inequality (2.21). The remaining part of the proof is quite analogous to the proof of Theorem 1, using now (3.11) or (3.13).

In order to complete the proof of Theorem 3, we give an example of function $\varphi$ satisfying (3.21)-(3.23).

$$
\varphi(x)= \begin{cases}\exp \left(\int_{x}^{0} \frac{a_{x}}{a} \mid d \sigma\right) \cdot \int_{x_{0}}^{x} \exp \left(\int_{0}^{\sigma}\left|\frac{a_{x}}{a}\right| d \tau\right) d \sigma, & 0 \leq x \leq x_{0}, \\ \exp \left(\int_{1}^{x} \frac{a_{x}}{a} \mid d \sigma\right) \cdot \int_{x_{0}}^{x} \exp \left(\int_{\sigma}^{1} \frac{a_{x}}{a} \mid d \tau\right) d \sigma, & x_{0} \leq x \leq 1,\end{cases}
$$


where $0<\mathrm{x}_{0}<1$ is the unique solution of the following transcendental equation

$$
a(1) \int_{x_{0}}^{0} \exp \left(\int_{0}^{\sigma} \frac{a_{x}}{a} \mid d \tau\right) d \sigma+a(0) \int_{x_{0}}^{1} \exp \left(\int_{\sigma}^{1} \frac{a_{x}}{a} \mid d \tau\right) d \sigma=0
$$

which is just the condition (3.23).

Remark 3.1 Similarly, we can also consider the sub-linear case. If we assume there exist some positive constants $\mathrm{C}_{1}, \mathrm{C}_{2}$ and $\mathrm{p}<1$ such that

$$
\mathrm{C}_{1}|\mathrm{~s}| \leq|\mathrm{g}(\mathrm{s})| \leq \mathrm{C}_{2} \operatorname{Max}(|\mathrm{s}|,|\mathrm{s}| \mathrm{p}), \quad \forall \mathrm{s} \in \mathbb{R}
$$

then given any constant $M>1$, there exists a constant $\mu>0$ depending on $E(0)$ such that the following "symetrical" estimate holds

$$
\mathrm{E}(\mathrm{t}) \leq \mathrm{ME}(0)(1+\mu \mathrm{t})^{-\frac{2 \mathrm{p}}{1-\mathrm{p}}}, \quad \forall \mathrm{t} \geq 0 .
$$

Starting from estimate (3.34), which is similar to (2.21), we have just to copy then the proof of Theorem 2.

Remark 3.2 Obviously, we can prove the same estimates when (3.2) is replaced by the slightly more general condition (for $\alpha$ and $\beta$ positive constants)

$$
\begin{cases}\operatorname{ay}_{x}(0, t)-\alpha y(0, t)=g\left(y^{\prime}(0, t)\right) & t>0 \\ \operatorname{ay}_{x}(1, t)+\beta y(1, t)=-g\left(y^{\prime}(1, t)\right) & t>0\end{cases}
$$

Remark 3.3 We can also replace the condition (3.2) by

$$
\begin{cases}\left(\operatorname{ay}_{x}\right)(0, t)=0, & t>0 \\ \left(\operatorname{ay}_{x}\right)(1, t)+\alpha y(1, t)=-g\left(y^{\prime}(1, t)\right), & t>0\end{cases}
$$

In that case, instead of the functional $\rho_{1}(t)$, we consider

$$
\hat{\rho}(t)=2 \int_{0}^{1} y^{\prime} \hat{\varphi} y_{x} d x+\hat{C}_{0} y(1, t) \int_{0}^{1} y^{\prime} d x
$$


where $\hat{C}_{0}$ is a positive constant and the function $\hat{\varphi}$ is defined hereafter by (3.44).

Accordingly, we consider the following energy

$$
\hat{\mathrm{E}}(\mathrm{t})=\frac{1}{2} \int_{0}^{1}\left(a\left|y_{x}\right|^{2}+\left|y^{\prime}\right|^{2}\right) \mathrm{dx}+\frac{\alpha}{2} y^{2}(1, \mathrm{t}) .
$$

A straightforward computation shows that

$$
\left|\hat{\rho}_{1}(t)\right| \leq \hat{C}_{3} \hat{E}(t)
$$

$$
\hat{\rho}_{1}^{\prime}(t) \leq-\hat{E}(t)+\hat{C}_{6}\left|y^{\prime}(1, t)\right|^{2}+\hat{C}_{7} g^{2}\left(y^{\prime}(1, t)\right)
$$

where $\hat{C}_{6}, \hat{C}_{7}$ are constants depending only on the variable coefficient a and $\hat{\varphi}$ is given by

$$
\hat{\phi}(x)=\exp \left(\int_{1}^{x} \frac{a_{x}}{a} \mid d \sigma\right) \cdot \int_{0}^{x} \exp \left(\int_{\sigma}^{1} \frac{a_{x}}{a} d \tau\right) d \sigma .
$$

Thus, we have proved that the estimates (3.12), (3.14) and (3.39) are still valid for any solution $y$ of the equations (3.1), (3.2)' and (3.3). 


\section{References}

[1] B. d'ANDREA-NOVEL, F. BOUSTANY and B.P. RAO, Control of an overhead crane: feedback stabilization of a hybrid PDE-ODE system, to appear.

[2] H. BREZIS, Maximal Monotone Operators, North Holland, Amsterdam, 1973.

[3] G. CHEN, Energy decay estimates and exact boundary value controllability for the wave equation in a boundary domain, J. Math. Pures Appl., 58 (1979), 249-274.

[4] G. CHEN and H. K. WANG, Asymptotic behavior of solutions of the one-dimensional wave equation with a nonlinear elastic dissipative boundary stabilizer, SIAM J. Contr. Opt., 22, 4 (1989), 758-775.

[5] C.M. DAFERMOS and M. SLEMROD, Asymptotic behavior of nonlinear contraction semigroups, J. Funct. Anal. 13 (1973), 97-106.

[6] P. GRISVARD, Contrôlabilité exacte avec des conditions mêlées, C.R. Acad. Sc. Paris Sér. I, 305 (1987), 363-366.

[7] P. GRISVARD, Contrôlabilité exacte des solutions de l'équation des ondes en présence de singularités, J. Math. Pures Appl., 68 (1989), 215-259.

[8] A. HARAUX, Semilinear hyperbolic problems in bounded domains, Mathematical Reports, Vol. 3, J. Dieudonné, ed. Harwood Academic Publishers, Gordon and Breach, New York, 1987.

[9] V. KOMORNIK, Contrôlabilité exacte en un temps minimal, C.R. Acad. Sc. Paris Sér.I, 304 (1987), 223-225.

[10] V. KOMORNIK, Exact controllability in short time for the wave equation, Ann. IHP, Analyse Nonlinéaire, 6 (1989), 152-164.

[11] V. KOMORNIK and E. ZUAZUA, Stabilisation frontière de l'équation des ondes: une méthode directe, C.R. Acad. Sc. Paris Sér. I, 305 ( 1987), 605-608.

[12] V. KOMORNIK and E. ZUAZUA, A direct method for the boundary stabilization of the wave equation, J. Math. Pures Appl., 69 (1990), 33-54.

[13] I. LASIECKA, Stabilization of wave equations with nonlinear dissipative damping on the boundary, in Proc. 26 $6^{\text {th }}$ IEEE Conference on Decision and Control, Los Angeles, CA, 1987, 2348-2349.

[14] I. LASIECKA, Stabilization of wave and plate-like equations with nonlinear dissipative damping on the boundary, Applied Mathematics Report \# RM-88-05, University of Virginia, Charlottesville VA, March 1988.

[15] J. LAGNESE, Decay of solutions of wave equations in a bounded region with boundary dissipation, J. Differential Equations, 50 (1983) 163-182.

[16] J.L. LIONS and E. MAGENES, Problemes aux limites non homegènes, Dunod, Paris, 1968. 
[17] M. SLEMROD, Feedback stabilization of a linear system in Hilbert space with an a priori bounded control, Math. Control Signals Systems, 2 (1989), 265-285.

[18] E. ZUAZUA, Some remarks on the boundary stabilizability of the wave equation, in Control of Boundaries and Stabilization, J. Simon, ed. Lecture Notes in Control and Information Sciences, Springer-Verlag, Berlin, New York, 1990.

[19] E. ZUAZUA, Uniform stabilization of the wave equation by nonlinear boundar feedback, SIAM J. Control Optim., 28 (1990), 466-477.

[20] E. ZUAZUA, Robustesse du feedback de stabilisation par contrôle frontière, C.R. Acad. Sc. Paris Sér. I, 307 ( 1988), 587-591. 
ISSN 0249 - 6399 